\title{
Administration de traitements anti-VIH et antirétroviraux en Afrique subsaharienne : de 2003 à 2017
}

\author{
G Taylor ${ }^{1 *}$
}

\section{Résumé}

Il faut tenir compte de plusieurs facteurs lorsqu'il est question de contrôler la pandémie du $\mathrm{VIH}$ et du SIDA en Afrique subsaharienne. Une combinaison de médicaments antirétroviraux est essentiel pour réduire la morbidité et la mortalité chez les personnes vivant avec le VIH et ce traitement a un rôle à jouer contre la transmission du VIH. Toutefois, I'accès aux traitements antirétroviraux d'association est très inégal à travers le monde, notamment en Afrique subsaharienne. Bien que les coûts liés aux traitements antirétroviraux d'association ne fassent plus obstacle à l'obtention d'un traitement efficace (ils coûtent actuellement moins de 100 \$ US par an), le nombre insuffisant de cliniciens qualifiés, l'absence de cliniques et de laboratoires, et l'augmentation de la prévalence de morbidités connexes (comme la tuberculose) compromettent la gestion du $\mathrm{VIH}$.

Un certain nombre d'initiatives ont été mises en application pour remédier à ce problème. L'une d'entre elles est la mise sur pied de l'Infectious Diseases Institute à l'Université de Makerere à Kampala (Ouganda). Cet institut a pour objectif d'offrir des soins cliniques aux personnes touchées par le $\mathrm{VIH}$, d'encourager la recherche clinique ayant trait à l'Afrique subsaharienne et à l'Ouganda en particulier, et d'offrir une formation clinique. Mon premier rôle était celui de formateur dans le cadre d'un programme de formation en traitements antirétroviraux et en gestion du VIH et du SIDA d'un grand nombre de cliniciens, ayant pour objectif d'en faire des dirigeants de programmes cliniques d'envergure dans leur société.

D'importants progrès ont été réalisés dans l'accessibilité aux traitements antirétroviraux d'association, et la mortalité due au VIH et au SIDA ainsi que les nouveaux cas d'infection diminuent. Néanmoins, si l'on souhaite atteindre les objectifs de l'Organisation mondiale de la santé (qui sont de 90-90-90) d'ici l'année 2020, il reste à étendre les services offerts et à élaborer de nouvelles approches à l'égard de la gestion du VIH. Outre l'offre de soins cliniques pratiques, les fournisseurs de soins de santé canadiens peuvent aider en transférant à des cliniciens locaux leurs compétences cliniques et en élaborant des paradigmes cliniques rationalisés et de nouvelles technologies permettant de gérer le VIH à long terme malgré un nombre limité de ressources.

\begin{abstract}
Affiliation
${ }^{1}$ Département de médecine, Université de l'Alberta, Edmonton (Alberta)
\end{abstract}

*Correspondance: geoff.taylor@ ualberta.ca

Citation proposée : Taylor G. Administration de traitements anti-VIH et antirétroviraux en Afrique subsaharienne : de 2003 à 2017. Relevé des maladies transmissibles au Canada. 2018;44(2):76-9. https://doi.org/10.14745/ ccdr.v44i02a06f

\section{Introduction}

Selon le Programme commun des Nations Unies sur le VIH/ SIDA, plus de 35 millions de personnes sont décédées à cause de troubles liés au VIH depuis I'apparition de l'épidémie du VIH et du SIDA dans les années 1980. En 2016, on estimait que, sur 36,7 millions de personnes vivant avec le VIH, 25 millions vivaient en Afrique subsaharienne. Cette année-là, on comptait 1,8 million de nouveaux cas et 1 million de décès dans le monde (1).

Les traitements antirétroviraux sont utilisés dans la prévention et le traitement de l'infection par le VIH depuis leur invention à la fin des années 1980. Depuis l'introduction du traitement antirétroviral d'association dans les pays à revenu élevé, la morbidité et la mortalité chez les personnes vivant avec le $\mathrm{VIH}$ ont été nettement réduites et les traitements antirétroviraux $d$ 'association sont actuellement indiqués pour toutes les personnes séropositives (2). En supprimant la réplication virale, les traitements antirétroviraux d'association ont pour résultat d'empêcher que les personnes séropositives infectent leurs partenaires intimes. Par conséquent, dans l'attente de l'élaboration d'autres interventions biomédicales comme un vaccin efficace, le traitement antirétroviral d'association joue un rôle très important dans la prévention du VIH (2).

Au début des années 2000, les traitements antirétroviraux $d$ 'association étaient très inégalement répartis dans le monde. Dans son discours liminaire pour I'International AIDS Society 
(IAS) qu'il a prononcé en 2000 à Durban (Afrique du Sud), Mark Wainberg, à l'époque président de l'IAS, déclarait que seulement 7000 personnes dans toute l'Afrique subsaharienne recevaient des traitements antirétroviraux d'association. En 2001, dans le but de remédier à la situation, un groupe de médecins de l'Infectious Disease Society of America se sont associés à des collègues de l'Université de Makerere à Kampala (Ouganda) pour créer I'Infectious Diseases Institute (IDI) de I'Université de Makerere (3). Cet institut a pour objectif d'offrir des soins cliniques aux personnes touchées par le VIH, d'encourager la recherche clinique ayant trait à l'Afrique subsaharienne et d'offrir une formation clinique.

Mon premier rôle était celui de formateur dans le cadre d'un programme de formation en traitements antirétroviraux et en gestion du VIH et du SIDA d'un grand nombre de cliniciens, ayant pour objectif d'en faire des dirigeants de programmes cliniques d'envergure dans leur société. À mesure que s'est développée l'expertise locale, les activités relatives à la formation sont passées sous la responsabilité de formateurs locaux. Depuis lors, je suis devenu un examinateur externe pour I'Université de Makerere, chargé de veiller au respect des normes.

La formation clinique offerte par I'IDI met en jeu une combinaison d'apprentissages didactiques et d'expériences cliniques avec des patients internes et des patients en consultation externe de Kampala et des villages environnants. Les élèves, pour la plupart des médecins diplômés en provenance de plusieurs pays subsahariens, participent au programme de formation pendant une période de deux à quatre semaines.

En 2016, I'IDI avait formé 19691 fournisseurs de soins de santé, y compris des médecins, des pharmaciens, des techniciens de laboratoire et des associés de recherche provenant de 28 pays d'Afrique (4).

\section{La situation actuelle}

Suivent quelques observations tout à fait personnelles sur l'évolution d'une stratégie pour aborder la gestion du VIH et du SIDA en Afrique subsaharienne.

\section{L'accès à des médicaments antirétroviraux ne constitue plus un enjeu majeur}

Au départ, les agents antirétroviraux, qui coûtaient plus de 10000 \$ US par an par personne, étaient hors de prix pour les patients et les programmes publics de l'Afrique subsaharienne (3). Un certain nombre de solutions ont été trouvées : les agents antirétroviraux génériques, produits hors licence de brevet, ont comblé partiellement le manque. Plus tard, les fabricants de marque ont autorisé les fabricants de médicaments génériques à produire une large gamme d'agents antirétroviraux. Actuellement, il est possible de se procurer un traitement antirétroviral d'association pour seulement 100 \$ US par an (5). Les donateurs sont d'une importance cruciale à l'apport d'un soutien aux programmes publics. Parmi les principaux donateurs, citons le Fonds mondial, qui dépense actuellement quatre milliards de dollars américains par année pour appuyer des programmes locaux de lutte contre le VIH, le SIDA, la tuberculose et le paludisme et, aux États-Unis, le President's Emergency Plan for AIDS Relief, un programme du gouvernement américain qui a donné depuis 2003 l'équivalent de soixante-douze milliards de dollars à des initiatives de soutien en matière de VIH et de SIDA. Grâce à ces initiatives, entre autres, les gouvernements africains ont pu augmenter progressivement la disponibilité de traitements antirétroviraux (1).

\section{Le manque de cliniciens formés en gestion du VIH constitue un énorme obstacle au traitement}

L'Ouganda, qui compte 41 millions d'habitants (le Canada en compte 36 millions), dispose de quatre écoles de médecine. II y en a 17 au Canada. En Ouganda, le ratio médecins-population est de 10 médecins pour 100000 habitants. Ce même ratio est de 228 médecins pour 100000 habitants au Canada. De même, le ratio infirmière-patient est très faible dans les unités de soins internes et l'on s'attend à ce que les patients soient accompagnés par un membre de la famille capable de fournir une grande partie des soins pratiques.

La plupart des cliniciens qui offrent des soins VIH sont des généralistes pour qui il convient d'offrir une approche simple et directe en ce qui concerne les traitements antirétroviraux. En règle générale, les cliniciens suivent les lignes directrices de l'Organisation mondiale de la santé (2) qui préconise une approche de santé publique plutôt qu'une approche plus spécialisée et plus complexe à l'égard des traitements.

Le salaire des médecins est typiquement très faible, même si ces spécialistes du milieu universitaire complètent leur maigre salaire par des consultations privées après les heures normales d'ouverture. II arrive que le salaire offert par les organisations non gouvernementales (ONG) soit plus élevé que celui offert par le secteur public, phénomène qui représente un enjeu important si les ONG souhaitent ne pas provoquer de distorsions sur le marché du travail médical en attirant les cliniciens de programmes publics en manque de personnel. Certains étudiants qui participent au programme de formation de I'IDI veulent se faire accréditer précisément dans le but d'améliorer leurs chances d'être embauchés par une ONG offrant un meilleur salaire.

\section{Enjeux cliniques}

Les fournisseurs de soins de santé en Afrique subsaharienne travaillent dans des conditions fortement défavorisées par rapport à leurs homologues de milieux à revenu élevé. Ils doivent affronter des défis liés à un nombre très élevé de patients $\mathrm{VIH}$ séropositifs et à un nombre croissant de personnes ayant besoin $d^{\prime}$ 'un traitement qui requiert un suivi étroit. En outre, le VIH ne représente qu'un trouble parmi tant d'autres nécessitant une intervention immédiate. Même si l'infection tuberculeuse accompagnée, ou non, du VIH est très répandue dans les unités de soins internes en Ouganda, on y manque malgré cela de tests de dépistage pour la tuberculose. En outre, il n'existe pas de chambres d'hôpital munies de systèmes de ventilation à pression négative. On ne trouve que très peu d'appareils respiratoires jetables N95 et, lorsqu'on en trouve, ils ont généralement déjà été utilisés. Par conséquent, il n'est pas inhabituel que l'infection 
tuberculeuse soit transmise aux patients et au personnel des unités de soins internes.

En ce qui concerne les soins ambulatoires, il est rare d'avoir accès à des infrastructures de laboratoires de dépistage et de suivi du VIH, notamment en ce qui concerne la concentration plasmatique du VIH dans I'ARN (à savoir la charge virale) dont le but est d'évaluer l'efficacité des traitements antirétroviraux (6). Au lieu de cela, les cliniciens examinent les patients beaucoup plus fréquemment qu'au Canada en cherchant des signes d'échec clinique. Ce faisant, ils imposent un fardeau supplémentaire aux patients et à l'infrastructure des cliniques. En cas d'échec clinique, il n'y a aucun moyen de vérifier la résistance aux antirétroviraux et les cliniciens sont obligés de procéder par estimation éclairée en ce qui concerne un traitement de rattrapage (2). Il existe un certain nombre d'agents antirétroviraux qui font obstacle à l'acquisition d'une résistance (7). En raison de leur coût, ces agents ne sont cependant pas disponibles actuellement sur les premières lignes de traitement en Afrique subsaharienne. S'il était possible de s'en procurer, ils pourraient offrir une solution au problème de la résistance aux médicaments antirétroviraux.

\section{Discussion}

Des avancées importantes ont été réalisées dans toute l'Afrique subsaharienne en ce qui concerne l'accessibilité de traitements antirétroviraux. En 2015, environ 17 des 37 millions de personnes séropositives du monde entier, dont 12 millions en Afrique subsaharienne, ont reçu des traitements (1). Les taux de mortalité dus aux $\mathrm{VIH}$ et au SIDA sont en baisse partout sur le continent. Toutefois, il reste encore beaucoup à faire. En 2016, environ 1,4 million d'Ougandais vivaient avec le VIH (par rapport à 75500 Canadiens en 2014) et l'on comptait 52300 nouveaux cas en Ouganda (par rapport à 2570 au Canada en 2014) (1,8). Chaque personne infectée est candidate à l'identification, à I'évaluation, aux conseils, aux traitements antirétroviraux, à un suivi à long terme et au maintien de ses soins.

\section{Que réserve l'avenir?}

Pour atteindre l'objectif fixé pour l'année 2020, soit 90-90-90 dans le cadre du Programme commun des Nations Unies sur le $\mathrm{VIH} / \mathrm{SIDA}$, visant à ce que $90 \%$ de toutes les personnes vivant avec le VIH connaissent leur statut, que $90 \%$ des personnes diagnostiquées aient accès à un traitement antirétroviral et que $90 \%$ de celles-ci soient virologiquement négatives) (2), la portée des programmes de traitements antirétroviraux devra être grandement élargie à toute l'Afrique subsaharienne. II ne faudra toutefois pas perdre le contact avec les patients recevant actuellement des traitements et sans contrôle virologique, ni le faire au détriment d'autres priorités relatives à la santé. Il s'agit d'une perspective intimidante. Reste à voir si le VIH pourrait être la première maladie virale contrôlée sans vaccin efficace. II faudrait élaborer des approches novatrices en matière de gestion, comme le fait de commencer les traitements antirétroviraux dès le premier contact. II faudrait corriger la situation relative à l'infrastructure des laboratoires. La numération des CD4 ne joue plus le rôle central qu'elle jouait à l'égard de l'admissibilité aux traitements. Le fait de pouvoir déterminer la charge virale au moyen de gouttes de sang séchées plutôt qu'au moyen de plasmas serait susceptible de faciliter grandement l'accès à des renseignements sur la charge virale, notamment dans les milieux situés loin des laboratoires de traitement. Bien que le fait d'utiliser des gouttes de sang séchées soit accompagné d'une certaine perte de sensibilité, il pourrait s'agir de la meilleure solution étant donné les moyens déjà insuffisants en ce qui concerne les tests de charge virale à travers l'Afrique subsaharienne $(6,9)$.

\section{Que peuvent faire les fournisseurs de soins de santé canadiens?}

Il existe plusieurs façons de participer aux activités visant le contrôle de la pandémie qui sévit en Afrique subsaharienne et dans d'autres milieux à faibles ressources. Il peut être très valorisant de fournir des soins directs aux patients et les soins cliniques directs de longue durée fournis par les cliniciens canadiens peuvent avancer les choses.

Pour les non-initiés, la pratique clinique en Afrique subsaharienne est très différente de celle du Canada. Les cliniques opèrent dans un milieu de grande activité et les ressources d'enquête sont souvent absentes, même dans les établissements hospitaliers de soins tertiaires. Les cliniciens doivent souvent adopter une démarche syndromique plutôt que diagnostique à l'égard de la gestion des patients. Il est essentiel d'avoir un certain degré de familiarité avec les activités liées à la gestion du VIH et du SIDA (y compris avec les traitements antirétroviraux d'association) bien qu'il ne soit pas nécessaire d'être un surspécialiste, et il serait également souhaitable d'avoir des connaissances liées à la gestion d'états pathologiques associés, notamment la tuberculose. Plutôt que de tenter d'élaborer un nouveau programme, je recommande vivement d'établir une relation avec une ONG existante disposant de solides antécédents. La clé de la réussite repose sur la durabilité de toute initiative.

Les fournisseurs de soins canadiens peuvent également aider d'autres manières. Les cliniciens, notamment les enseignants-cliniciens qui connaissent bien le $\mathrm{VIH}$, le SIDA et les états pathologiques associés, peuvent transmettre leurs compétences cliniques aux fournisseurs de soins locaux. Les techniciens de laboratoire, les analystes du domaine des services de santé et les consultants en politiques de santé peuvent offrir des conseils sur l'élaboration de paradigmes cliniques rationalisés et sur de nouvelles technologies visant la gestion à long terme du $\mathrm{VIH}$ dans des contextes où les ressources sont limitées.

D'importants progrès ont été réalisés en ce qui concerne la gestion de la pandémie du VIH/SIDA en Afrique subsaharienne. Cependant, il reste de nombreux défis à relever et de nombreuses occasions d'aider s'offrent aux fournisseurs de services de santé canadiens.

\section{Conflit d'intérêt}

Aucun. 
5. ONUSIDA. Communiqué de presse : Plus de 6 millions de personnes reçoivent maintenant un traitement contre le VIH en Afrique subsaharienne. Genève : Le Programme commun des Nations Unies sur le VIH/SIDA ; le 6 juillet 2012. http:// www.unaids.org/fr/resources/presscentre/pressreleaseandsta tementarchive/2012/july/20120706prafricatreatment

6. Lecher S, Williams J, Fonjungo PN, Kim AA, Ellenberger $D$, Zhang $G$ et al. Progress with Scale-Up of HIV Viral Load Monitoring - Seven Sub-Saharan African Countries, January 2015-June 2016. MMWR Morb Mortal Wkly Rep 2016 Dec;65(47):1332-5. DOl (http://dx.doi.org/10.15585/ mmwr.mm6547a2). PubMed (https://www.ncbi.nlm.nih. gov/entrez/query.fcgi?cmd=Retrieve\&db=PubMed\&lis t_uids=27906910\&dopt=Abstract).

7. Panel on Antiretroviral Guidelines for Adults and Adolescents. Guidelines for the use of antiretroviral agents in adults and adolescents living with HIV. Washington (DC): US Department of Health and Human Services; 2017. http:// www.aidsinfo.nih.gov/ContentFiles/AdultandAdolescentGL. pdf

8. Agence de la santé publique du Canada. Le VIH et le sida au Canada - Rapport de surveillance en date du 31 décembre 2014. Ottawa (ON): ASOC; 2015. https://www.canada.ca/fr/ sante-publique/services/publications/maladies-et-affections/ vih-et-sida-canada-rapport-surveillance-31-decembre-2014. html

9. Johannessen A, Garrido C, Zahonero N, Sandvik L, Naman E, Kivuyo SL et al. Dried blood spots perform well in viral load monitoring of patients who receive antiretroviral treatment in rural Tanzania. Clin Infect Dis 2009 Sep;49(6):976-81. DOI (http://dx.doi.org/10.1086/605502). PubMed (https://www. ncbi.nlm.nih.gov/entrez/query.fcgi?cmd=Retrieve\&db=PubM ed\&list_uids=19663598\&dopt=Abstract). 